\title{
Numerical verification of topological crossings in band structure of solids
}

\author{
Geunsik Lee ${ }^{1}$, Jai Sam Kim ${ }^{1}$ and J Zak ${ }^{2}$ \\ ${ }^{1}$ Department of Physics, Pohang University of Science and Technology (POSTECH), \\ Pohang 790-784, Republic of Korea \\ 2 Department of Physics, Technion-Israel Institute of Technology, Haifa 32000, Israel
}

Received 23 October 2002, in final form 18 February 2003

Published 17 March 2003

Online at stacks.iop.org/JPhysCM/15/2005

\begin{abstract}
There have been recent predictions of topologically unavoidable branch crossing in the energy band structure of solids. In this paper calculations of energy dispersion relations are carried out in a number of crystals with orthorhombic space group (SG) symmetry. Our calculations verify the topological crossings and are in good agreement with the predictions based on representations of SGs.
\end{abstract}

\section{Introduction}

The notion of accidental degeneracy in solids was first introduced by Herring in 1937 [1]. According to Herring, accidental degeneracy is "not necessitated by the symmetry and reality of the Hamiltonian'. As is well known, the reality of the Hamiltonian was used by Wigner for defining time reversal symmetry [2]. A degeneracy in the band structure of solids is understood as a crossing or touching of two or more energy branches at a given point $\vec{k}$ in the Brillouin zone (BZ). The symmetry labelling of Bloch functions was started in a classical paper by Bouckaert et al [3] on the basis of irreducible representations (irreps) of space groups (SGs). This labelling was followed up by Herring to include time reversal, which uses the reality of the Hamiltonian [4]. SG symmetry has also been used for symmetry labelling of Wannier functions [5-7], which has eventually led to the definition of the band representations of SGs [8]. Unlike an irrep of an SG which is labelled by symmetries of different $k$-vectors in the BZ, a band representation assigns a symmetry label to entire energy bands, and thus enables one to carry out a global analysis of them. This feature of globality was recently used for showing that there exist topologically unavoidable crossings of energy branches in the band structure of solids [9]. These crossings are similar to the accidental degeneracy predicted by Herring [1] in that they are not necessitated by the symmetry and reality of the Hamiltonian. However, they are also very different in the following sense. Accidental degeneracy depends on the detailed functional dependence of the potential energy (and not only on its symmetry) and can be removed by changing the form of the potential without changing its symmetry. On the other hand, the topologically unavoidable branch crossing is a consequence of symmetry and global 
continuity of the Bloch functions in their dependence on the $\vec{k}$-vector in the BZ. The location in $\vec{k}$-space of the topological crossing can be moved by changing the potential, but it cannot be removed as long as the symmetry of the crystal is kept unchanged.

In this paper we present examples of topologically unavoidable energy branch crossings in a great variety of crystals with orthorhombic SG symmetry. These crossings were recently predicted on the basis of band representation analysis [10] in combination with continuity chords [8]. The numerical results are presented in the form of energy dispersion relations in a number of directions in the BZ. The crossings usually occur between two branches and they can appear in one or more directions in the BZ. In [10] rules were established for predicting the topological crossings, and in this paper our numerical results confirm these crossings and enable us to associate each crossing with the rule that leads to it. The main attention is paid in this paper to branch crossings in crystals with orthorhombic non-symmorphic SG symmetry. As was shown in [10] there are a number of orthorhombic SGs which should exhibit topological crossings. We confirm this prediction by carrying out a band structure calculation for some actual crystals with orthorhombic SG symmetry.

\section{Identification of representations}

Depending on the desired physical properties one can choose extended Bloch functions or localized Wannier functions for the bases of electronic wavefunctions. Bloch functions have been widely used in band calculations whereas Wannier functions have been revived due to their advantage for some local phenomena such as impurities, surface effects, polarizations etc.

Most electronic structure calculation methods, LAPW, LMTO, OPW, KKR and so on, are based on Bloch functions and use two kinds of basis for eigenstates, depending on the sharpness of the crystal potential. In the atomic core region, where the potential is very sharp, spherical harmonics are used for the solutions of the radial Schrödinger equation. In the interstitial region between atoms, where the potential is smooth, the plane wave basis is used. In order to determine the SG symmetry properties of eigenstates, it suffices to analyse the wavefunction in the interstitial region.

For our numerical works we used two methods, the FLAPW method [11] and the pseudopotential method [12]. Both methods are based on the density functional theory employing the local density approximation for the exchange-correlation energy functional. They differ in the way of treating the potentials due to the nuclei and the core electrons but they both use plane wave expansions to approximate the wavefunctions in the interstitial region. The eigenstate $\psi_{n \vec{k}}$, where $n$ is the band index and $\vec{k}$ is a reciprocal vector in the first BZ, is expressed in the plane wave basis in the following form:

$$
\psi_{n \vec{k}}=\sum_{\vec{K}} c_{n}(\vec{K}) \mathrm{e}^{\mathrm{i} \vec{k} \cdot \vec{r}} \exp (\mathrm{i} \vec{K} \cdot \vec{r})
$$

where $\vec{K}$ is a reciprocal lattice vector. The summation is over all reciprocal lattice vectors, the number of which is infinite in theory but is cut off to a finite number in numerical calculations.

The symmetry of the Hamiltonian $\mathcal{H}$ of a crystal is specified in the way the atoms are positioned in the lattice. By specifying the atomic positions in the unit cell in a Bravais lattice we determine the SG $G$ of the crystal. Since the Hamiltonian $\mathcal{H}$ commutes with the operations of the SG $G$ the eigenstates are often degenerate and transform like a representation vector of $G$. Thus the symmetry of the wavefunction is specified by identifying its SG representation. In order to find which representation of the little group $G_{\vec{k}}$ of $\vec{k}$ the calculated eigenstates carry, 
Table 1. Crystal structures for six materials.

\begin{tabular}{|c|c|c|c|}
\hline Crystal & SG & Lattice parameter $(\AA)$ & Atomic position \\
\hline $\mathrm{Ag}_{2} \mathrm{Se}$ & $19 P 2{ }_{1} 2_{1} 2_{1}$ & $a=4.333, b=7.062, c=7.764$ & $\begin{array}{l}\mathrm{Ag}_{1}(0.357,0.619,0.456) \\
\mathrm{Ag}_{2}(0.978,0.279,0.361) \\
\mathrm{Se}(0.608,0.485,0.149)\end{array}$ \\
\hline AsGeSe & 52 Pnna & $a=5.062, b=10.117, c=11.687$ & $\begin{array}{l}\text { As }(0.0781,0.4148,0.1282) \\
\mathrm{Ge}_{1}(0.25,0.0,0.7640) \\
\mathrm{Ge}_{2}(0.2873,0.25,0.25) \\
\mathrm{Se}(0.9288,0.8435,0.1091)\end{array}$ \\
\hline $\mathrm{OPb}$ & $57 \mathrm{Pbcm}$ & $a=5.891, b=5.489, c=4.775$ & $\begin{array}{l}\mathrm{O}(0.8691,0.0886,0.25) \\
\mathrm{Pb}(0.2309,0.9792,0.25)\end{array}$ \\
\hline $\mathrm{CMo}_{2}$ & $60 \mathrm{Pbcn}$ & $a=4.732, b=6.037, c=5.204$ & $\begin{array}{l}C_{1}(0.0,0.355,0.25) \\
C_{2}(0.0,0.866,0.25) \\
\text { Mo }(0.249,0.130,0.083)\end{array}$ \\
\hline $\mathrm{PdSe}_{2}$ & $61 \mathrm{Pbca}$ & $a=5.741, b=5.866, c=7.691$ & $\begin{array}{l}\operatorname{Pd}(0.0,0.0,0.0) \\
\operatorname{Se}(0.112,0.117,0.407)\end{array}$ \\
\hline $\mathrm{BFe}$ & 62 Pnma & $a=5.506, b=2.952, c=4.061$ & $\begin{array}{l}\mathrm{B}(0.031,0.25,0.620) \\
\mathrm{Fe}(0.180,0.25,0.125)\end{array}$ \\
\hline
\end{tabular}

we use the following projection identity [2]:

$$
\phi_{i}=\sum_{R} \chi_{i}(R) R \psi_{n \vec{k}},
$$

where $\psi_{n \vec{k}}$ is the computed wavefunction, $R$ is a group operation which corresponds to an element of the little group $G_{\vec{k}}$ and $\chi_{i}(R)$ is the character of the $R$ operation in the $i$ th representation of $G_{\vec{k}}$. Since $\psi_{n \vec{k}}$ is symmetrized with respect to the little group operations, $\psi_{n \vec{k}}$ belongs to one of the irreps of $G_{\vec{k}}$. If $\psi_{n \vec{k}}$ does not belong to the $i$ th irrep, $\phi_{i}$ is zero due to the orthogonality between two different representations. If $\psi_{n \vec{k}}$ belongs to an irrep, only one of $\phi_{i}$ is non-zero, while the others are zero.

We computed energy bands of six materials, $\mathrm{Ag}_{2} \mathrm{Se}$, AsGeSe, $\mathrm{OPb}, \mathrm{CMo}_{2}, \mathrm{PdSe}_{2}$ and BFe. Their crystal structures are given in table 1. All information is taken from Pearson's handbook [13]. Kohn-Sham equations [14] were solved with the FLAPW method [11], where self-consistency was achieved until averaged charge difference within the atomic sphere (determined by $R_{M T}$ ) becomes less than 0.001 electrons. All parameters in computation for the six crystals are given in table 2 . $R_{M T}$ is the muffin-tin radius for each atom in atomic units. The LDA for the exchange-correlation potential $\left(V_{X C}\right)$ is that of Perdew and Wang [15]. The GGA for $V_{X C}$ is that of Perdew et al [16]. $R * K_{\max }$ is the smallest of all atomic radii multiplied by the plane wave cut-off of the wavefunction in atomic unit. $l$ is the maximum angular quantum number of partial waves used inside atomic spheres. $G_{\max }$ is the plane wave cut-off of charge density in atomic unit. In the last column, the number of mesh points along each primitive axis of the orthohombic BZ is given as $\left(n_{1} \times n_{2} \times n_{3}\right)$ and the number of inequivalent $\vec{k}$ points out of $n_{1} n_{2} n_{3}$ points is specified. We used the FLAPW method implemented by the WIEN group [17] and double-checked the results with the pseudo-potential method implemented by the FHI group [18].

Let us take an example. The crystal BFe has the orthohombic structure. Its SG number is $62(P n m a)$. Its band structure is illustrated in figure 6 below. We consider the special points $\Gamma, \mathrm{X}$ and $\mathrm{Y}$ in the BZ. Characters of irreps of the little groups at points $\Gamma, \mathrm{X}$ and $\mathrm{Y}$ are given in table 3(a)-(c) [19,20]. Inserting the characters given in table 3 into equation (2), it is straightforward to find irreps of the eigenstates $\psi_{n \vec{k}}$, at each $\vec{k}$ point. 
Table 2. Parameters used in the FLAPW computation.

\begin{tabular}{lllllll}
\hline Crystal & $R_{M T}(\mathrm{au})$ & $V_{X C}$ & $R * K_{\max }$ & $l$ & $G_{\max }$ & $\vec{k}$ points \\
\hline $\mathrm{Ag}_{2} \mathrm{Se}$ & Ag: $2.4, \mathrm{Se}: 2.3$ & LDA & 7.0 & 10 & 10.0 & $8(3 \times 2 \times 2)$ \\
$\mathrm{AsGeSe}$ & As: $2.2, \mathrm{Ge}: 2.2, \mathrm{Se}: 2.2$ & LDA & 6.0 & 10 & 10.0 & $6(4 \times 2 \times 1)$ \\
$\mathrm{OPb}$ & O: $2.0, \mathrm{~Pb}: 2.0$ & GGA & 8.0 & 10 & 10.0 & $27(4 \times 4 \times 5)$ \\
$\mathrm{CMo}_{2}$ & C: $1.75, \mathrm{Mo}: 2.0$ & GGA & 7.0 & 10 & 10.0 & $27(5 \times 4 \times 4)$ \\
$\mathrm{PdSe}_{2}$ & Pd: $2.25, \mathrm{Se}: 2.2$ & LDA & 7.0 & 10 & 10.0 & $18(4 \times 4 \times 3)$ \\
$\mathrm{BFe}$ & B: $1.7, \mathrm{Fe}: 2.0$ & GGA & 8.0 & 10 & 10.0 & $45(4 \times 8 \times 5)$ \\
\hline
\end{tabular}

Table 3. (a) Irreps of SG 62 (Pnma, $\mathrm{D}_{2 \mathrm{~h}}^{16}$ ) (taken from [19]). (a) Irreps at $\vec{k}=(0,0,0) . E$ is the identity and $I$ is the inversion operation. $U^{x}$ means twofold rotation about the $x$ axis. $\sigma^{x}$ means reflection by a plane normal to the $x$ axis. (b) Irreps at $\vec{k}=\left(\frac{\pi}{a}, 0,0\right)$. (c) Irreps at $\vec{k}=\left(0, \frac{\pi}{b}, 0\right)$.

\begin{tabular}{|c|c|c|c|c|c|c|c|c|}
\hline \multicolumn{9}{|l|}{ (a) } \\
\hline$\Gamma$ & $\Gamma_{1}$ & $\Gamma_{2}$ & $\Gamma_{3}$ & $\Gamma_{4}$ & $\Gamma_{5}$ & $\Gamma_{6}$ & $\Gamma_{7}$ & $\Gamma_{8}$ \\
\hline$E$ & 1 & 1 & 1 & 1 & 1 & 1 & 1 & 1 \\
\hline$U^{x}$ & 1 & 1 & -1 & -1 & 1 & 1 & -1 & -1 \\
\hline$U^{y}$ & 1 & -1 & 1 & -1 & 1 & -1 & 1 & -1 \\
\hline$U^{z}$ & 1 & -1 & -1 & 1 & 1 & -1 & -1 & 1 \\
\hline$I$ & 1 & 1 & 1 & 1 & -1 & -1 & -1 & -1 \\
\hline$\sigma^{x}$ & 1 & 1 & -1 & -1 & -1 & -1 & 1 & 1 \\
\hline$\sigma^{y}$ & 1 & -1 & 1 & -1 & -1 & 1 & -1 & 1 \\
\hline$\sigma^{z}$ & 1 & -1 & -1 & 1 & -1 & 1 & 1 & -1 \\
\hline \multicolumn{9}{|l|}{ (b) } \\
\hline$X$ & $X_{1}$ & $\mathrm{X}_{2}$ & & & & & & \\
\hline$E$ & 2 & 2 & & & & & & \\
\hline$\left(\sigma^{y} \mid 0 \frac{b}{2} 0\right)$ & 2 & -2 & & & & & & \\
\hline \multicolumn{9}{|l|}{ (c) } \\
\hline $\mathrm{Y}$ & $\mathrm{Y}_{1}$ & $\mathrm{Y}_{2}$ & & & & & & \\
\hline$E$ & 2 & 2 & & & & & & \\
\hline$\left(\sigma^{z} \mid \frac{a}{2} 0 \frac{c}{2}\right)$ & 2 & -2 & & & & & & \\
\hline
\end{tabular}

Having identified the irrep of the eigenstates, we can go on to form the basis functions for the irrep. We use the method of projection operators given in [21],

$$
f_{j l}=\sum_{R} M(R)_{j l}^{*} R \psi_{n \vec{k}}
$$

The $j$ th partner in a set of basis functions, $M(R)_{j l}$ is the matrix element of the operator $R$ between the $j$ th and the $l$ th basis functions. Each $l$ gives a different set of basis functions. Let us take an example $\mathrm{X}_{1}$. The two-dimensional irrep is generated by

$$
\begin{array}{ll}
M\left(U^{x} \mid \frac{1}{2} \frac{1}{2} \frac{1}{2}\right) \equiv\left(\begin{array}{cc}
0 & -1 \\
1 & 0
\end{array}\right), & M\left(U^{z} \mid \frac{1}{2} 0 \frac{1}{2}\right) \equiv\left(\begin{array}{cc}
1 & 0 \\
0 & -1
\end{array}\right), \\
M\left(\sigma^{x} \mid \frac{1}{2} \frac{1}{2} \frac{1}{2}\right) \equiv\left(\begin{array}{cc}
1 & 0 \\
0 & -1
\end{array}\right) .
\end{array}
$$

Using equation (3) we obtain two sets of two-dimensional bases for $\mathrm{X}_{1}$ :

$$
\begin{aligned}
u_{1}^{(1)}(x, y, z)= & 2\left\{\exp \left(\mathrm{i} 2 \pi\left(n_{x}+\frac{1}{2}\right) x\right)+\mathrm{i}(-1)^{m} \exp \left(-\mathrm{i} 2 \pi\left(n_{x}+\frac{1}{2}\right) x\right)\right\} \mathrm{e}^{\mathrm{i} 2 \pi n_{z} z} \\
& \times \cos \left(2 \pi n_{y} y\right) \quad \text { for } n_{y}=\text { even } \\
& \times \sin \left(2 \pi n_{y} y\right) \quad \text { for } n_{y}=\text { odd },
\end{aligned}
$$




$$
\begin{array}{rlr}
u_{2}^{(1)}(x, y, z)= & 2\left\{-\exp \left(-\mathrm{i} 2 \pi\left(n_{x}+\frac{1}{2}\right) x\right)+\mathrm{i}(-1)^{m} \exp \left(\mathrm{i} 2 \pi\left(n_{x}+\frac{1}{2}\right) x\right)\right\} \mathrm{e}^{-\mathrm{i} 2 \pi n_{z} z} \\
& \times \cos \left(2 \pi n_{y} y\right) \quad \text { for } n_{y}=\text { even } \\
& \times(-1) \sin \left(2 \pi n_{y} y\right) \quad \text { for } n_{y}=\text { odd; } \\
u_{1}^{(2)}(x, y, z)= & 2\left\{-\exp \left(-\mathrm{i} 2 \pi\left(n_{x}+\frac{1}{2}\right) x\right)-\mathrm{i}(-1)^{m} \exp \left(\mathrm{i} 2 \pi\left(n_{x}+\frac{1}{2}\right) x\right)\right\} \mathrm{e}^{-\mathrm{i} 2 \pi n_{z} z} \\
& \times \cos \left(2 \pi n_{y} y\right) \quad \text { for } n_{y}=\text { even } \\
& \times(-1) \sin \left(2 \pi n_{y} y\right) \quad \text { for } n_{y}=\text { odd, } \\
u_{2}^{(2)}(x, y, z)= & 2\left\{\exp \left(\mathrm{i} 2 \pi\left(n_{x}+\frac{1}{2}\right) x\right)-\mathrm{i}(-1)^{m} \exp \left(-\mathrm{i} 2 \pi\left(n_{x}+\frac{1}{2}\right) x\right)\right\} \mathrm{e}^{\mathrm{i} 2 \pi n_{z} z} \\
& \times \cos \left(2 \pi n_{y} y\right) \quad \text { for } n_{y}=\operatorname{even} \\
& \times \sin \left(2 \pi n_{y} y\right) \quad \text { for } n_{y}=\text { odd, }
\end{array}
$$

where $m=n_{x}-n_{z}+n_{y}, \exp \left(\mathrm{i} k_{x} \frac{a}{2}\right)=\exp (\mathrm{i} \pi / 2)=\mathrm{i}$. The wavefunction for $\mathrm{X}_{1}$ computed by whatever method should be expressible in terms of the above bases.

Using equation (3) we obtain one-dimensional bases for $\Gamma_{1}, \Gamma_{3}, \Gamma_{8}$ and $\Gamma_{6}$ : $v_{1}(x, y, z)=2\left\{\exp \left(\mathrm{i} 2 \pi n_{x} x\right)+(-1)^{m} \exp \left(-\mathrm{i} 2 \pi n_{x} x\right)\right\} \mathrm{e}^{\mathrm{i} 2 \pi n_{z} z}$

$$
\begin{array}{rrr} 
& \times \cos \left(2 \pi n_{y} y\right) & \text { for } n_{y}=\text { even } \\
& \times \sin \left(2 \pi n_{y} y\right) & \text { for } n_{y}=\text { odd } \\
+ & 2\left\{\exp \left(-\mathrm{i} 2 \pi n_{x} x\right)+(-1)^{m} \exp \left(\mathrm{i} 2 \pi n_{x} x\right)\right\} \mathrm{e}^{-\mathrm{i} 2 \pi n_{z} z} \\
& \times \cos \left(2 \pi n_{y} y\right) & \text { for } n_{y}=\text { even } \\
\times(-1) \sin \left(2 \pi n_{y} y\right) & \text { for } n_{y}=\text { odd, }
\end{array}
$$

$v_{3}(x, y, z)=2\left\{\exp \left(\mathrm{i} 2 \pi n_{x} x\right)-(-1)^{m} \exp \left(-\mathrm{i} 2 \pi n_{x} x\right)\right\} \mathrm{e}^{\mathrm{i} 2 \pi n_{z} z}$

$$
\begin{aligned}
& \times \cos \left(2 \pi n_{y} y\right) \quad \text { for } n_{y}=\text { even } \\
& \times \sin \left(2 \pi n_{y} y\right) \quad \text { for } n_{y}=\text { odd } \\
& +2\left\{\exp \left(-\mathrm{i} 2 \pi n_{x} x\right)-(-1)^{m} \exp \left(\mathrm{i} 2 \pi n_{x} x\right)\right\} \mathrm{e}^{-\mathrm{i} 2 \pi n_{z} z} \\
& \times \cos \left(2 \pi n_{y} y\right) \quad \text { for } n_{y}=\text { even } \\
& \times(-1) \sin \left(2 \pi n_{y} y\right) \quad \text { for } n_{y}=\text { odd, }
\end{aligned}
$$

$v_{8}(x, y, z)=2\left\{\exp \left(\mathrm{i} 2 \pi n_{x} x\right)+(-1)^{m} \exp \left(-\mathrm{i} 2 \pi n_{x} x\right)\right\} \mathrm{e}^{\mathrm{i} 2 \pi n_{z} z}$

$$
\begin{array}{rrr} 
& \times \cos \left(2 \pi n_{y} y\right) \quad & \text { for } n_{y}=\text { even } \\
& \times \sin \left(2 \pi n_{y} y\right) \quad & \text { for } n_{y}=\text { odd } \\
- & 2\left\{\exp \left(-\mathrm{i} 2 \pi n_{x} x\right)+(-1)^{m} \exp \left(\mathrm{i} 2 \pi n_{x} x\right)\right\} \mathrm{e}^{-\mathrm{i} 2 \pi n_{z} z} \\
& \times \cos \left(2 \pi n_{y} y\right) & \text { for } n_{y}=\text { even } \\
& \times(-1) \sin \left(2 \pi n_{y} y\right) & \text { for } n_{y}=\text { odd, }
\end{array}
$$

$$
\begin{aligned}
v_{6}(x, y, z)= & 2\left\{\exp \left(\mathrm{i} 2 \pi n_{x} x\right)-(-1)^{m} \exp \left(-\mathrm{i} 2 \pi n_{x} x\right)\right\} \mathrm{e}^{\mathrm{i} 2 \pi n_{z} z} \\
& \times \cos \left(2 \pi n_{y} y\right) \quad \text { for } n_{y}=\text { even } \\
& \times \sin \left(2 \pi n_{y} y\right) \quad \text { for } n_{y}=\text { odd } \\
- & 2\left\{\exp \left(-\mathrm{i} 2 \pi n_{x} x\right)-(-1)^{m} \exp \left(\mathrm{i} 2 \pi n_{x} x\right)\right\} \mathrm{e}^{-\mathrm{i} 2 \pi n_{z} z} \\
& \times \cos \left(2 \pi n_{y} y\right) \quad \text { for } n_{y}=\text { even } \\
& \times(-1) \sin \left(2 \pi n_{y} y\right) \quad \text { for } n_{y}=\text { odd },
\end{aligned}
$$

where $m=n_{x}-n_{z}+n_{y}$.

Since $k_{x}=0$ at the $\Gamma$-point we can obtain $v_{k}(x, y, z)$ from $u_{i}^{(j)}(x, y, z)$ by replacing $\frac{1}{2}$ with zero and $i$ with unity. With this recipe we get $u_{1}^{(1)}-u_{1}^{(2)} \rightarrow v_{1}, u_{1}^{(1)}+u_{1}^{(2)} \rightarrow v_{8}$, $u_{2}^{(2)}-u_{2}^{(1)} \rightarrow v_{3}$ and $u_{2}^{(2)}+u_{2}^{(1)} \rightarrow v_{6}$. 
In the same way as for $\mathrm{X}_{1}$, we obtain two sets of two-dimensional bases for $\mathrm{Y}_{1}$ and $\mathrm{Y}_{2}$ :

$$
\begin{aligned}
& w_{1}^{(1)}(x, y, z)=\left[\mathrm{e}^{\mathrm{i}(\alpha+\beta)}+(-1)^{n_{y}}(-1)^{m} \mathrm{e}^{-\mathrm{i}(\alpha+\beta)}\right] \mathrm{e}^{\mathrm{i} \gamma} \\
& +\left[\mathrm{e}^{-\mathrm{i}(\alpha+\beta)}+(-1)^{n_{y}}(-1)^{m} \mathrm{e}^{\mathrm{i}(\alpha+\beta)}\right] \mathrm{e}^{-\mathrm{i} \gamma} \quad \text { for } Y_{1} \\
& -\left[\mathrm{e}^{-\mathrm{i}(\alpha+\beta)}+(-1)^{n_{y}}(-1)^{m} \mathrm{e}^{\mathrm{i}(\alpha+\beta)}\right] \mathrm{e}^{-\mathrm{i} \gamma} \quad \text { for } Y_{2} \text {, } \\
& w_{2}^{(1)}(x, y, z)=\mathrm{i}\left[(-1)^{m} \mathrm{e}^{\mathrm{i}(\alpha-\beta)}+(-1)^{n_{y}} \mathrm{e}^{-\mathrm{i}(\alpha-\beta)}\right] \mathrm{e}^{-\mathrm{i} \gamma} \\
& +\mathrm{i}\left[(-1)^{m} \mathrm{e}^{-\mathrm{i}(\alpha-\beta)}+(-1)^{n_{y}} \mathrm{e}^{\mathrm{i}(\alpha-\beta)}\right] \mathrm{e}^{\mathrm{i} \gamma} \quad \text { for } Y_{1} \\
& -\mathrm{i}\left[(-1)^{m} \mathrm{e}^{-\mathrm{i}(\alpha-\beta)}+(-1)^{n_{y}} \mathrm{e}^{\mathrm{i}(\alpha-\beta)}\right] \mathrm{e}^{\mathrm{i} \gamma} \quad \text { for } Y_{2} \text {, } \\
& w_{1}^{(2)}(x, y, z)=\mathrm{i}\left[(-1)^{m} \mathrm{e}^{\mathrm{i}(\alpha-\beta)}-(-1)^{n_{y}} \mathrm{e}^{-\mathrm{i}(\alpha-\beta)}\right] \mathrm{e}^{-\mathrm{i} \gamma} \\
& -\mathrm{i}\left[(-1)^{m} \mathrm{e}^{-\mathrm{i}(\alpha-\beta)}-(-1)^{n_{y}} \mathrm{e}^{\mathrm{i}(\alpha-\beta)}\right] \mathrm{e}^{\mathrm{i} \gamma} \quad \text { for } Y_{1} \\
& +\mathrm{i}\left[(-1)^{m} \mathrm{e}^{-\mathrm{i}(\alpha-\beta)}-(-1)^{n_{y}} \mathrm{e}^{\mathrm{i}(\alpha-\beta)}\right] \mathrm{e}^{\mathrm{i} \gamma} \quad \text { for } Y_{2}, \\
& w_{2}^{(2)}(x, y, z)=\left[\mathrm{e}^{\mathrm{i}(\alpha+\beta)}-(-1)^{n_{y}}(-1)^{m} \mathrm{e}^{-\mathrm{i}(\alpha+\beta)}\right] \mathrm{e}^{\mathrm{i} \gamma} \\
& -\left[\mathrm{e}^{-\mathrm{i}(\alpha+\beta)}-(-1)^{n_{y}}(-1)^{m} \mathrm{e}^{\mathrm{i}(\alpha+\beta)}\right] \mathrm{e}^{-\mathrm{i} \gamma}, \quad \text { for } Y_{1} \\
& +\left[\mathrm{e}^{-\mathrm{i}(\alpha+\beta)}-(-1)^{n_{y}}(-1)^{m} \mathrm{e}^{\mathrm{i}(\alpha+\beta)}\right] \mathrm{e}^{-\mathrm{i} \gamma}, \quad \text { for } Y_{2},
\end{aligned}
$$

where $\exp \left(\mathrm{i} k_{y} \frac{b}{2}\right)=\exp (\mathrm{i} \pi / 2)=\mathrm{i}, \alpha=2 \pi n_{x} x, \beta=2 \pi\left(n_{y}+\frac{1}{2}\right) y$ and $\gamma=2 \pi n_{z} z$.

Once again we can obtain $v_{k}(x, y, z)$ from $w_{i}^{(j)}(x, y, z)$ by replacing $\frac{1}{2}$ with zero and $i$ with unity. We get $w_{1}^{(1)}+w_{2}^{(1)} \rightarrow v_{1}, w_{1}^{(2)}+w_{2}^{(2)} \rightarrow v_{6}$ for $\mathrm{Y}_{1}$ and $w_{1}^{(1)}-w_{2}^{(1)} \rightarrow v_{8}$, $w_{2}^{(2)}-w_{1}^{(2)} \rightarrow v_{3}$ for $\mathrm{Y}_{2}$.

While a Bloch state is an energy eigenstate for a particular wavevector $\vec{k}$, a Wannier function describes an energy band as a whole. Wannier functions (generalized Wannier functions for composite bands) form bases of a band representation. Whereas the conventional irrep of an SG $G$ is induced from a small representation of the little group of a wavevector $\vec{k}$, $G_{\vec{k}} \subseteq G$, an irreducible elementary band representation is induced from a small irrep of the little group of the Wyckoff position $\vec{w}, G_{\vec{w}} \subseteq G$.

Given the SG and the Wyckoff position $\vec{w}$ one finds the number of branches $b(\vec{w}, \rho)$ of an elementary band representation (a full classification of elementary band representations was given in [22]) according to the formula (see $[9,10])$

$$
b(\vec{w}, \rho)=\left[\operatorname{dim} D^{(\vec{w}, \rho)}\right] \frac{|P|}{\left|G_{\vec{w}}\right|},
$$

where $\left[\operatorname{dim} \mathrm{D}^{(\vec{w}, \rho)}\right]$ is the dimension of the irrep $D^{(\vec{w}, \rho)}$ of the little group $G_{\vec{w}}$ of the Wyckoff position $\vec{w}$ and $|P|$ and $\left|G_{\vec{w}}\right|$ are the numbers of elements of the group $P$ (the point group of the SG) and $G_{\vec{w}}$. In table 5 we list those Wyckoff positions which correspond to the lowest energy band in each solid. For all of them $\operatorname{dim} \mathrm{D}^{(\vec{w}, \rho)}=1$. For SG no $19\left(P 2_{1} 2_{1} 2_{1}\right)$ the point group is $\mathrm{D}_{2} \equiv 222$, and the little group $G_{\vec{w}}$ is the unit element only. For all other cases chosen in table 5 the point group $P$ is $\mathrm{D}_{2 \mathrm{~h}} \equiv \mathrm{mmm}$, and the little group $G_{\vec{w}}$ consists of two elements, the unit element and a rotation by $\pi$, a reflection in a plane or the inversion (see table 5). For all of them, $|P|=8$ and $\left|G_{\vec{w}}\right|=2$. From equation (17) and table 5 it then follows that all the elementary energy bands that we consider have four branches.

Now let us investigate how the four branches of the elementary band associated with a Wyckoff position are grouped into a single family. From table 4 there are four Wyckoff positions in SG number 62, three special positions and one general position. Three positions, whose labels are $(a),(b)$ and $(c)$, have two symmetry operations that leave each position invariant up to equivalence. Wyckoff positions $(a)$ and $(b)$ have an inversion symmetry $\overline{1}$ and (c) has $\left(\sigma^{y} \mid 0 \frac{b}{2} 0\right)$-symmetry. 
Table 4. Wyckoff positions and their symmetries of SG 62 (taken from [25]).

\begin{tabular}{|c|c|c|c|}
\hline Multiplicity & Wyckoff letter & Site symmetry & Coordinates \\
\hline 8 & $\mathrm{~d}$ & 1 & $\begin{array}{l}\begin{array}{lll}\text { (1) } x, y, z & \text { (2) } \bar{x}+\frac{1}{2}, \bar{y}, z+\frac{1}{2} & \text { (3) } \bar{x}, y+\frac{1}{2}, \bar{z}\end{array} \\
\begin{array}{lll}\text { (4) } x+\frac{1}{2}, \bar{y}+\frac{1}{2}, \bar{z}+\frac{1}{2} & \text { (5) } \bar{x}, \bar{y}, \bar{z} & \text { (6) } x+\frac{1}{2}, y, \bar{z}+\frac{1}{2} \\
\text { (7) } x, \bar{y}+\frac{1}{2}, z & \text { (8) } \bar{x}+\frac{1}{2}, y+\frac{1}{2}, z+\frac{1}{2}\end{array}\end{array}$ \\
\hline 4 & $\mathrm{c}$ &. $\mathrm{m}$ & $x, \frac{1}{4}, z \quad \bar{x}+\frac{1}{2}, \frac{3}{4}, z+\frac{1}{2} \quad \bar{x}, \frac{3}{4}, \bar{z} \quad x+\frac{1}{2}, \frac{1}{4}, \bar{z}+\frac{1}{2}$ \\
\hline 4 & $\mathrm{~b}$ & $\overline{1}$ & $0,0, \frac{1}{2} \quad \frac{1}{2}, 0,0 \quad 0, \frac{1}{2}, \frac{1}{2} \quad \frac{1}{2}, \frac{1}{2}, 0$ \\
\hline 4 & a & $\overline{1}$ & $0,0,0 \quad \frac{1}{2}, 0, \frac{1}{2} \quad 0, \frac{1}{2}, 0 \quad \frac{1}{2}, \frac{1}{2}, \frac{1}{2}$ \\
\hline
\end{tabular}

Table 5. Listing of crystals with corresponding symmetries for which energy band calculations are carried out in this paper. The information in the first seven columns is explained in the headings. The explanation of the last two columns is given in the text.

\begin{tabular}{|c|c|c|c|c|c|c|}
\hline SG & $\begin{array}{l}\text { Point Wyckoff } \\
\text { group position }\end{array}$ & $\begin{array}{l}\text { Isotropy } \\
\text { group }\end{array}$ & $\begin{array}{l}\text { Band } \\
\text { representation }\end{array}$ & Crystal & Rule & $\begin{array}{l}\text { Symmetry elements } \\
\text { and directions in } \\
\text { BZ }\end{array}$ \\
\hline $19 P 2{ }_{1} 2_{1} 2_{1}$ & $222 \quad \mathrm{a}(x y z)$ & $E$ & $(\mathrm{a}, 1)$ & $\mathrm{Ag}_{2} \mathrm{Se}$ & $2 b$ & $U^{x}(\mathrm{X}), U^{y}(\mathrm{Y}), U^{z}(\mathrm{Z})$ \\
\hline 52 Pnna & $m m m$ c $\left(\frac{a}{4} 0 z\right)$ & $C_{2}^{z}$ & $(c, 2)$ & AsGeSe & 1 & $\sigma^{z}(\mathrm{X}), \sigma^{z}(\mathrm{Y})$ \\
\hline $57 \mathrm{Pbcm}$ & $m m m \mathrm{~d}\left(x y \frac{c}{4}\right)$ & $C_{s}^{z}$ & $(\mathrm{~d}, 1)$ & $\mathrm{OPb}$ & $2 \mathrm{a}$ & $\sigma^{x}(Z), \sigma^{z}(Y)$ \\
\hline 60 Pbcn & $m m m \mathrm{c}\left(0 y \frac{c}{4}\right)$ & $C_{2}^{y}$ & $(\mathrm{c}, 1)$ & $\mathrm{CMO}_{2}$ & $2 \mathrm{a}$ & $\sigma^{x}(Z), U^{y}(\mathrm{Y})$ \\
\hline $61 P b c a$ & $m m m \mathrm{~b}\left(00 \frac{c}{2}\right)$ & $C_{i}$ & $(b, 1)$ & $\mathrm{PdSe}_{2}$ & $2 \mathrm{a}$ & $\sigma^{x}(Z), \sigma^{z}(\mathrm{Y})$ \\
\hline 62 Pnma & $m m m \mathrm{c}\left(x \frac{b}{4} z\right)$ & $C_{s}^{y}$ & $(\mathrm{c}, 1)$ & $\mathrm{BFe}$ & $2 \mathrm{a}$ & $\sigma^{z}(\mathrm{Y}), \sigma^{y}(\mathrm{X})$ \\
\hline
\end{tabular}

In equation (17), $|P|=8,\left|G_{\vec{w}}\right|=2$ and $\left[\operatorname{dim} \mathrm{D}^{(\vec{w}, \rho)}\right]=1$ for each Wyckoff position $a, b$ and $c$. The elementary band is composed of four branches. In figure 6 there is one elementary band which has branches with the irreps $\Gamma_{1}, \Gamma_{3}, \Gamma_{6}$ and $\Gamma_{8}$ at the $\Gamma$ point and $\mathrm{X}_{1}$ at the $\mathrm{X}$ point. From the symmetries of these eigenstates at the $\Gamma$ and $X$ points, we can determine the symmetry of the band, that is the symmetry of the Wannier function. In the character table 3(a), $\Gamma_{1}, \Gamma_{3}, \Gamma_{6}$ and $\Gamma_{8}$ are even under $\left(\sigma^{y} \mid 0 \frac{b}{2} 0\right)$. In the case of the $\mathrm{X}$ point, $\mathrm{X}_{1}$ has even parity under $\left(\sigma^{y} \mid 0 \frac{b}{2} 0\right)$. Therefore all branches of the elementary band have the even parity under $\left(\sigma^{y} \mid 0 \frac{b}{2} 0\right)$. If we denote such a kind of band as $(c, 1)$, the band structure in figure 6 consists of one $(c, 1)$ elementary band.

\section{Unavoidable branch crossing examples and comparison with the rules}

With this information at hand one can now easily see how one predicts topological branch crossing for the SGs in table $5[9,10]$. In the centre of the BZ $\Gamma$, all the irreps of these SGs are one-dimensional, because their point groups are Abelian. On the other hand, on the surface of the BZ there is a number of $\vec{k}$-vectors at which the energy is doubly degenerate [19]. In order to follow in more detail how the branch crossing comes about, let us denote the symmetries of the four branches at $\Gamma$ by $\Gamma_{i}, i=1,2,3,4$ in the order of growing energy. Also, assume that at the points $\mathrm{X}$ and $\mathrm{Y}$ on the surface of the $\mathrm{BZ}$ we have two-dimensional representations only. We denote them by $\mathrm{X}_{1}, \mathrm{X}_{2}$ and by $\mathrm{Y}_{1}, \mathrm{Y}_{2}$. By using the notion of continuity chords [8] one can follow the symmetry of the branches when going from the centre $\Gamma$ of the BZ to the 


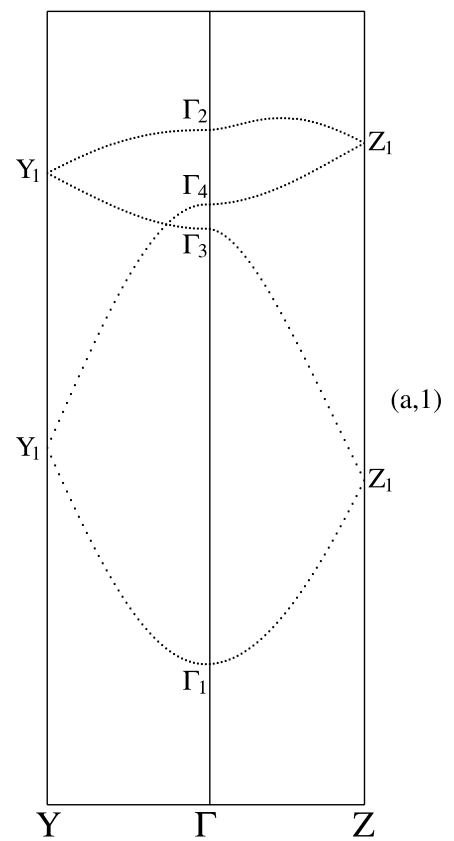

Figure 1. Energy curves for the lowest four-branch energy band in $\mathrm{Ag}_{2} \mathrm{Se}$ in the $y$ - and $z$-directions. $\Gamma, \mathrm{Y}$ and $\mathrm{Z}$ are symmetry points in the BZ. $\Gamma_{1}, \Gamma_{2}, \Gamma_{3}$ and $\Gamma_{4}$ label the one-dimensional irreps of SG no $19 P 2_{1} 2_{1} 2_{1}$ in the centre of the $\mathrm{BZ} \Gamma$. $\mathrm{Y}_{1}$ and $\mathrm{Z}_{1}$ are two-dimensional irreps of $P 2_{1} 2_{1} 2_{1}$ on the surface of the BZ [19]. The branch crossing is in the $y$-direction.

surface. As an example, let us assume that by symmetry and continuity the energy dispersion curves that start at the $\Gamma$-point as $\Gamma_{1}$ and $\Gamma_{2}$ have to join at the surface where they become together $\mathrm{X}_{1}$, and that in the $Y$-direction $\Gamma_{1}$ and $\Gamma_{3}$ have to come together at $\mathrm{Y}_{1}$. It is easy to see that this will necessarily lead to an unavoidable crossing in either the $X$ - or $Y$-direction in the BZ. A detailed derivation of such unavoidable crossings, including rules for their appearance is given in [10].

We turn now to our numerical results for the materials in table 5. As mentioned above we calculate the energy dispersion relations for the crystals in table 5. These crystals were chosen from [23] which lists different materials that crystallize according to a given SG symmetry. We find that all the lowest lying energy bands for the crystals in table 5 have four branches and that there is a crossing of these branches at least in one direction in the BZ as predicted. The results of our computations are presented in figures 1-6, where we give only the lowest energy band for each crystal. In all cases of figures 1-6 the next energy band is separated from the lowest band by an energy gap.

In [10] two rules were established for predicting what we call topologically unavoidable crossings in the band structure of solids. Rule 1 says that if there exists a symmetry element which has different characters in two different directions in BZ, then in one of these directions there is necessarily a crossing. One can check that rule 1 applies to the four-branch energy band in figure 2 for the crystal AsGeSe. The last two columns of table 5 list the rules that apply for predicting the crossing. For the Pnna SG symmetry the character of the $\sigma^{z}$-element (reflection in the plane perpendicular to the $z$-axis) at the X-point on the surface of the BZ (see [19]) is $\chi\left(\sigma^{z}\right)= \pm 2$; it is +2 for $X_{1}$ and -2 for $X_{2}$ (see figure 2). One can check that $\sigma^{z}$ is a symmetry element in the $X$ - and $Y$-directions of the BZ. But its character at the surface of 


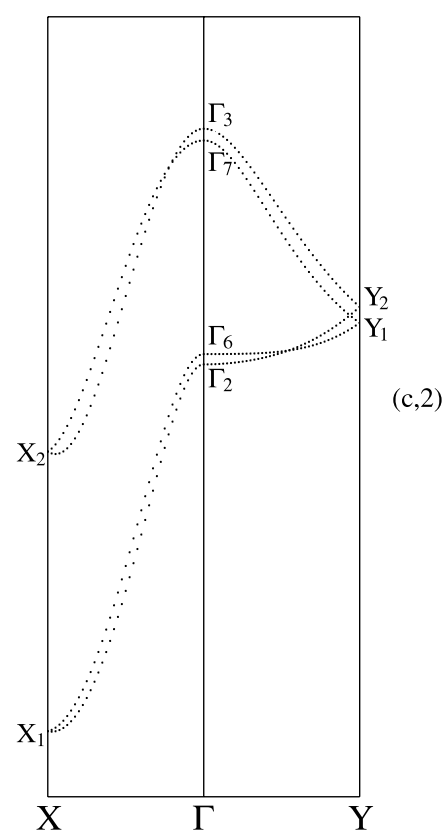

Figure 2. The same as in figure 1 but for the crystal AsGeSe in $x$ - and $y$-directions of the BZ with SG no 52 Pnna. Here the topologically unavoidable crossing is in the $y$-direction. The crossing in the figure in the $x$-direction is not required by symmetry and topology.

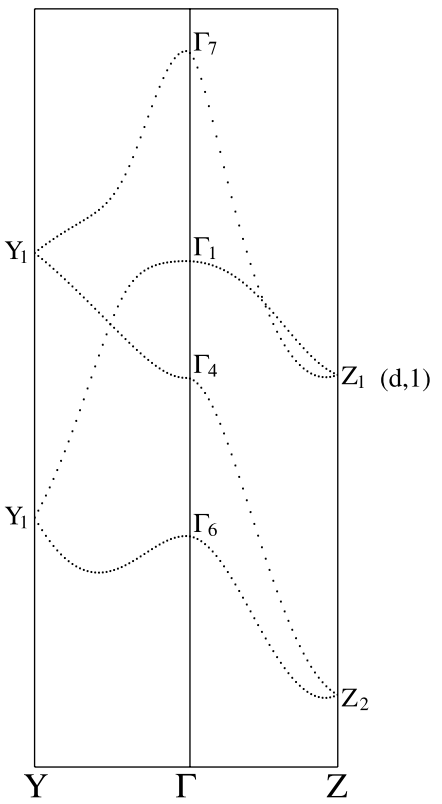

Figure 3. The same as in figure 1 but for the crystal $\mathrm{OPb}$ with SG no $57 \mathrm{Pbcm}$. The topologically unavoidable crossing is in the $y$-direction. The crossing in the figure in the $z$-direction is not required by symmetry and topology. 


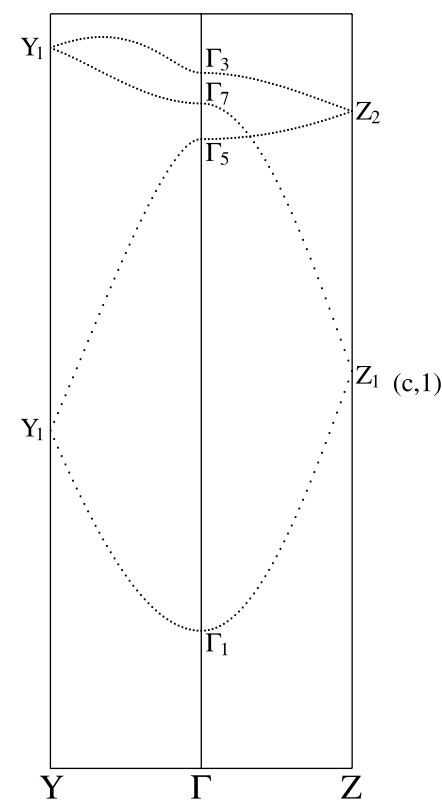

Figure 4. The same as in figure 1 but for the crystal $\mathrm{CMo}_{2}$ with $\mathrm{SG}$ no $60 \mathrm{Pbcn}$. The topologically unavoidable crossing is in the $z$-direction.

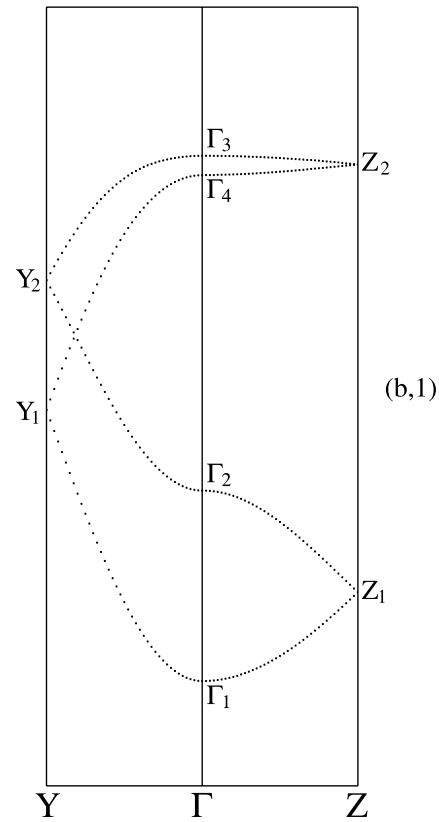

Figure 5. The same as in figure 1 but for the crystal $\mathrm{PdSe}_{2}$ with $\mathrm{SG}$ no $61 \mathrm{Pbca}$. The topologically unavoidable crossing is in the $y$-direction.

the BZ at $\mathrm{Y}$ is zero, $\chi\left(\sigma^{z}\right)=0$ (see figure 2). The characters of the irreps of SGs can be found in [19]. The last two columns of table 5 list rule 1 for the SG 52 and the symmetry element $\sigma^{z}$ for the $X$ - and $Y$-directions. 


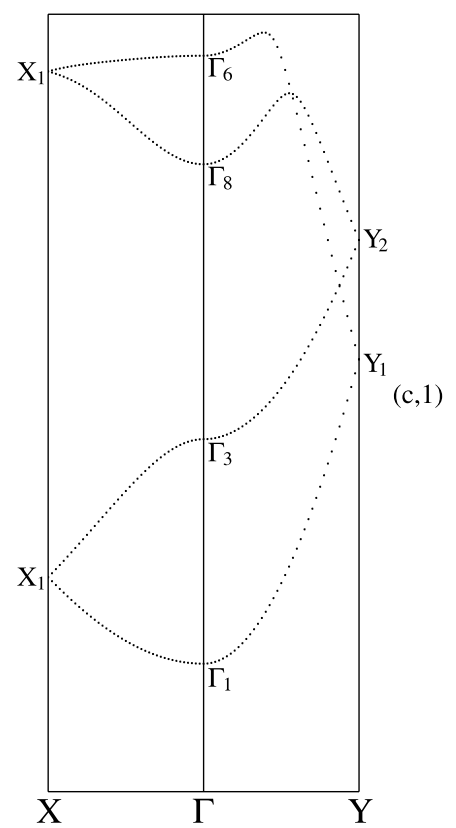

Figure 6. The same as in figure 2 but for the crystal BFe with SG no 62 Pnma. There are two topologically unavoidable crossings in the $y$-direction.

Rule 2 for branch crossings in [10] consists of two parts, $2 \mathrm{a}$ and $2 \mathrm{~b}$.

Rule 2a. If there exist two symmetry elements which have characters equal to \pm 2 in two different directions in the $\mathrm{BZ}$, then in one of these directions there is necessarily a crossing.

Rule 2b. If there exist three symmetry elements which have characters zero in two or three different directions in the BZ, then in one of these directions there is necessarily a crossing.

One can check that rule $2 \mathrm{~b}$ applies to the four-branch band in figure 1 for the crystal $\mathrm{Ag}_{2} \mathrm{Se}$, and that rule $2 \mathrm{a}$ applies to the four-branch bands in figures $3,4,5$ and 6 , respectively for the crystals $\mathrm{OPb}, \mathrm{CMo}_{2}, \mathrm{PdSe}_{2}$ and $\mathrm{BFe}$. This is shown in table 5, in the column before last. In the last column of table 5 we list the symmetry elements and the directions in the BZ (in the parentheses) which leads to the prediction of the crossings. Thus, for SG no 19 we list in the last column of table 5 the elements $U^{x}, U^{y}, U^{z}$ whose characters are zero (rule $2 \mathrm{~b}$ ). For the groups 57, 60, 61 and 62 rule 2a applies. It should be pointed out that the SGs in table 5 all have different Wyckoff positions (the $(c, 1)$ Wyckoff positions for SGs 60 and 62 are different despite their identical notation) and different little groups, and nevertheless the lowest four-branch energy band exhibits branch crossings. This feature of branch crossing was predicted in [10] and our calculations therefore confirm this prediction. Among the listing in table 5 is SG no 62 with the international symbol Pnma. In [23] it is pointed out that there are 794 inorganic crystals that crystallize with the Pnma symmetry. We claim that all these crystals should be expected to have topologically unavoidable branch crossings. An example of such a band crossing for GeS with the Pnma SG symmetry is found in [24].

\section{Conclusion}

In conclusion, our calculations confirm topological band crossings in the band structure of crystals having the symmetry of six non-symmorphic orthorhombic SGs (see table 5). 
According to [24], there are more than 1000 inorganic crystals and more than 1100 organic crystals that crystallize with the symmetry of these six SGs. This actually means that the phenomenon of topological branch crossing is highly abundant in nature. We have considered in detail the orthorhombic SGs, but topological branch crossing is not restricted to them only, and should exist in crystals with other SG symmetry. One should expect that the topological crossing will have an influence on the density of states of the crystal. Since the crossing point of the branches can be moved by changing the potential energy without changing its symmetry, application of pressure to the crystal can cause changes in the density of states in the BZ.

\section{Acknowledgments}

One of the authors (JZ) would like to thank Professor J Genossar and Dr G Reisner for valuable discussions, and the Department of Physics at Pohang University of Science and Technology (POSTECH) for their kind hospitality. This work is partially funded by the POSTECH fund for Basic Sciences.

\section{References}

[1] Herring C 1937 Phys. Rev. 52365

[2] Wigner E P 1959 Group Theory and its Application to the Quantum Mechanics of Atomic Spectra (New York: Academic)

[3] Bouckaert L P, Smoluchowski R and Wigner E P 1936 Phys. Rev. 5058

[4] Herring C 1937 Phys. Rev. 52361

[5] Burneika I P and Levinson I B 1961 Tr. Akad. Lit. SSR B4 31

[6] des Cloizeaux J 1963 Phys. Rev. 129554

[7] Kovalev O V 1975 Fiz. Tverd. Tela 171700 (Engl. transl. 1975 Sov. Phys.-Solid State 17 1106)

[8] Zak J 1980 Phys. Rev. Lett. 251025

Zak J 1982 Phys. Rev. B 251344

Zak J 1982 Phys. Rev. B 263010

[9] Michel L and Zak J 2000 Europhys. Lett. 50519 Michel L and Zak J 1999 Phys. Rev. 595988

[10] Zak J 2002 J. Phys. A: Math. Gen. 356509

[11] Wimmer E, Krakauer H, Weinert M and Freeman A J 1981 Phys. Rev. B 24864 and references therein Weinert M, Wimmer E and Freeman A J 1982 Phys. Rev. B 264571

[12] Cohen M L and Heine V 1970 Solid State Physics vol 24 (New York: Academic) p 38 Heine V and Weaire 1970 Solid State Physics vol 24 (New York: Academic) p 249

[13] Pearson W B 1985 Pearson's Handbook of Crystallographic Data for Intermetallic Phases (Metals Park, Ohio: American Society for Metals)

[14] Hohenberg P and Kohn W 1964 Phys. Rev. 136 B864 Kohn W and Sham L J 1965 Phys. Rev. 140 A1133

[15] Perdew J P and Wang Y 1992 Phys. Rev. B 4513244

[16] Perdew J P, Burke S and Ernzerhof M 1996 Phys. Rev. Lett. 773865

[17] Blaha P, Schwartz K and Luits J 1999 WIEN97, a Full Potential Linearized Augmented Plane Wave Package for Calculating Crystal Properties Technical University of Vienna (ISBN 3-9501031-0-4)

This is an improved and updated Unix version of the original copyrighted WIEN code, which was published by Blaha P, Schwartz K, Sorantin P and Trickey S B 1990 Comput. Phys. Commun. 59399

[18] Bockstedte M, Kley A, Neugebauer J and Scheffler M 1997 Comput. Phys. Commun. 107187

[19] Zak J, Casher A, Gluck M and Gur Y 1969 The Irreducible Representations of Space Groups ed J Zak (New York: Benjamin)

[20] Cracknell A P, Davies B L, Miller S C and Love W F 1979 Kronecker Product Tables vol 1 (New York: Plenum)

[21] Slater J C 1965 Symmetry and Energy Bands in Crystals (New York: Dover)

[22] Bacry M, Michel L and Zak J 1988 Springer Lecture Notes in Physics vol 313 (New York: Springer) p 291

[23] Mighell A D, Ondik H M and Molino B B 1977 J. Chem. Ref. Data 6675

[24] Grandke T and Ley L 1977 Phys. Rev. B 16832

[25] Hahn T (ed) 1996 International Tables for Crystallography vol A, Space Group Symmetry 4th revised edn (Dordrecht: Kluwer) 